Acta Crystallographica Section A

Foundations of Crystallography

ISSN 0108-7673

Received 6 January 2012

Accepted 26 April 2012

C) 2012 International Union of Crystallography Printed in Singapore - all rights reserved

\section{Phyllotaxis: a non-conventional crystalline solution to packing efficiency in situations with radial symmetry}

\author{
J.-F. Sadoc, ${ }^{a *}$ N. Rivier ${ }^{b}$ and J. Charvolin ${ }^{a}$ \\ aLaboratoire de Physique des Solides, Université Paris-Sud, CNRS UMR 8502, F-91405 Orsay \\ cedex, France, and ${ }^{\mathbf{b}}$ IPCMS, Université Louis Pasteur, F-67084 Strasbourg cedex, France. \\ Correspondence e-mail: sadoc@lps.u-psud.fr
}

\begin{abstract}
Phyllotaxis, the search for the most homogeneous and dense organizations of small discs inside a large circular domain, was first developed to analyse arrangements of leaves or florets in plants. It has since become an object of study not only in botany, but also in mathematics, computer simulations and physics. Although the mathematical solution is now well known, an algorithm setting out the centres of the small discs on a Fermat spiral, the very nature of this organization and its properties of symmetry remain to be examined. The purpose of this paper is to describe a phyllotactic organization of points through its Voronoi cells and Delaunay triangulation and to refer to the concept of defects developed in condensed matter physics. The topological constraint of circular symmetry introduces an original inflation-deflation symmetry taking the place of the translational and rotational symmetries of classical crystallography.
\end{abstract}

\section{Introduction}

The densest organization for a packing of small discs on an infinite plane is obtained when their centres are at the nodes of the triangular tiling of a hexagonal crystalline lattice; they all occupy the same area on this plane. If the discs are to be organized within a finite compact domain of the plane, this solution stays valid only if the boundaries of this domain are aligned along crystallographic directions. In the case of a domain with a circular border, a constant area per centre can only be obtained when the centres are regularly placed on the spiral drawn by the algorithm of phyllotaxis.

Tiling of surfaces such as a circular domain of the plane, a cylinder or a sphere according to phyllotaxis leads to the building of distributions of points having the best homogeneity and isotropy possible. This solution appears in plant growth as being the only one compatible with a sequential growth within the frame of a self-organizing process submitted to geometrical constraints (Bravais \& Bravais, 1837; Jean, 1983, 1992; Turing, 1992; Coxeter, 1961, 1972). This had been noticed by D'Arcy Thompson (1917) who wrote: ' . . and not the least curious feature of the case is the limited, even the small number of possible arrangements which we observe and recognize.'

This solution also appears in the formation of BénardMarangoni convection cells in cylindrical containers (Rivier et al., 1984; Rivier, 1992), the organization of ferrofluid droplets falling down in silicone oil in the presence of an inhomogeneous magnetic field with cylindrical symmetry (Douady \& Couder, 1992) and that of air bubbles on a circular water surface (Yoshikawa et al., 2010). Finally, spherical phyllotaxis was used to estimate the Earth coverage of satellite constellations (Gonzàlez, 2010).

A phyllotactic structure on a surface is a set of points that define the position of physical objects such as discs, convection cells, florets or others. We go into this matter describing a phyllotactic organization of points of the plane through its Voronoi cells and Delaunay triangulation and making reference to the concept of defects developed in condensed-matter physics. The topological constraint of circular symmetry introduces an original inflation-deflation symmetry taking the place of the translational and rotational symmetries of classical crystallography.

The phyllotactic structure is represented in Figs. 1, 2 and 3. Outside a core, it consists of grains of hexagonal cells (in red) which are concentric circular rings, bounded and separated by circular rings of defects (often also called grain boundaries) made of $n_{2}$ heptagonal cells (inner), $n_{1}$ hexagonal cells (middle) and $n_{2}$ pentagonal cells (outer). The two numbers $n_{1}$ and $n_{2}$ are successive Fibonacci numbers. These grain boundaries serve as natural boundaries for our optimal packing problem. Their properties which are presented here therefore solved the optimal packing problem topologically and metrically.

At the origin of our work are questions raised by the structure of collagen fibrils. They can be considered as dense packings with circular sections of more-or-less parallel rods, the so-called triple helices, whose lateral organization was the object of several investigations through X-ray scattering studies. These observations could not agree with a purely 


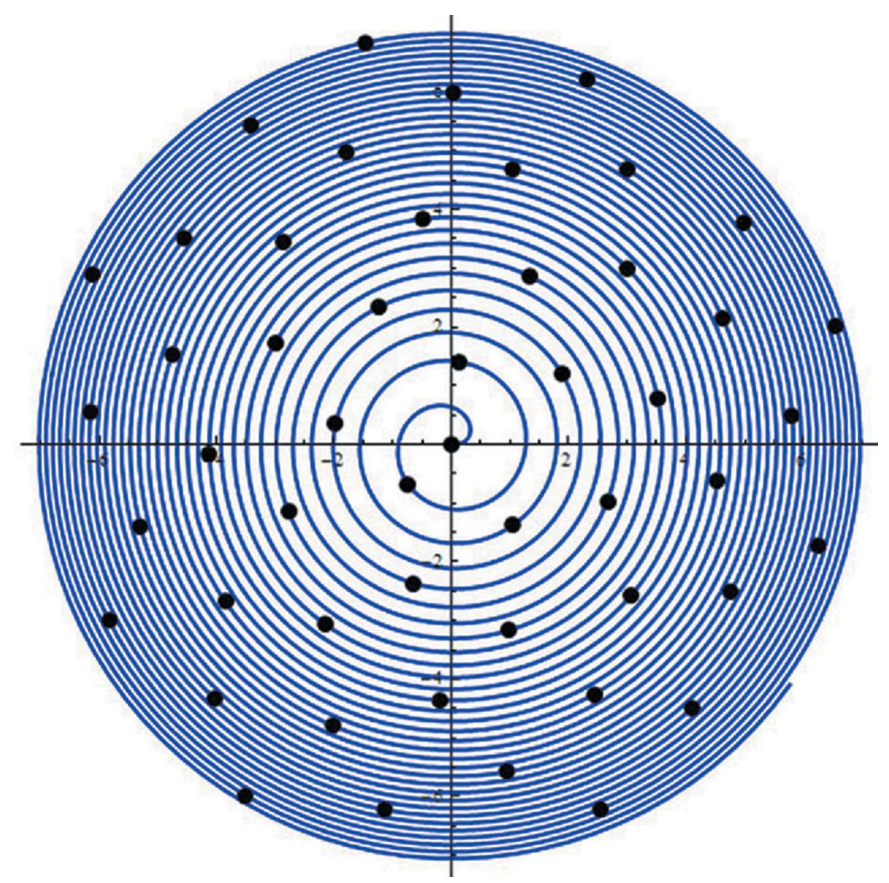

Figure 1

A Fermat spiral with 50 points on it, successively placed at angle $\theta=2 \pi s \lambda$, with $s=0$ to 49 .

hexagonal lattice and revealed the presence of an important degree of disorder. A lattice built from a distorted hexagonal lattice with a large unit cell, a quasicrystalline structure and paracrystalline fluctuations in a hexagonal lattice were then

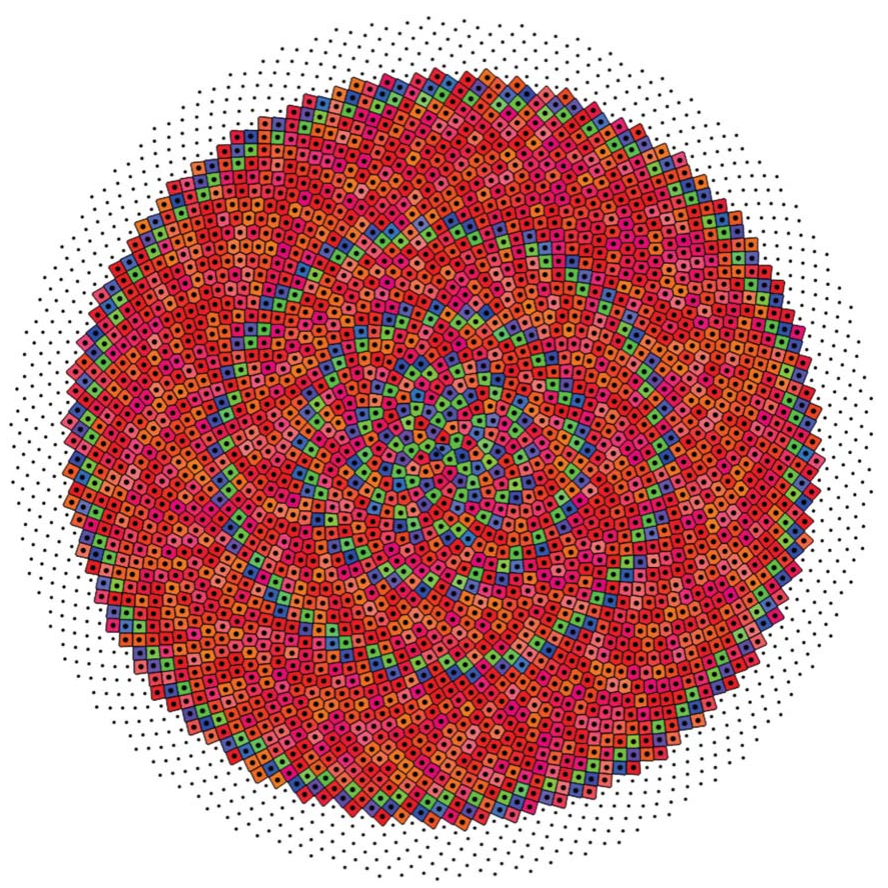

Figure 2

Voronoi decomposition of a 2500 -site model. Voronoi cells define the number of neighbours: six for hexagonal cells in red, five for pentagonal cells in blue and seven for heptagonal cells in green. Small black points are for sites over 2500 added in order to avoid difficulties encountered in defining Voronoi cells at the border of a finite set.

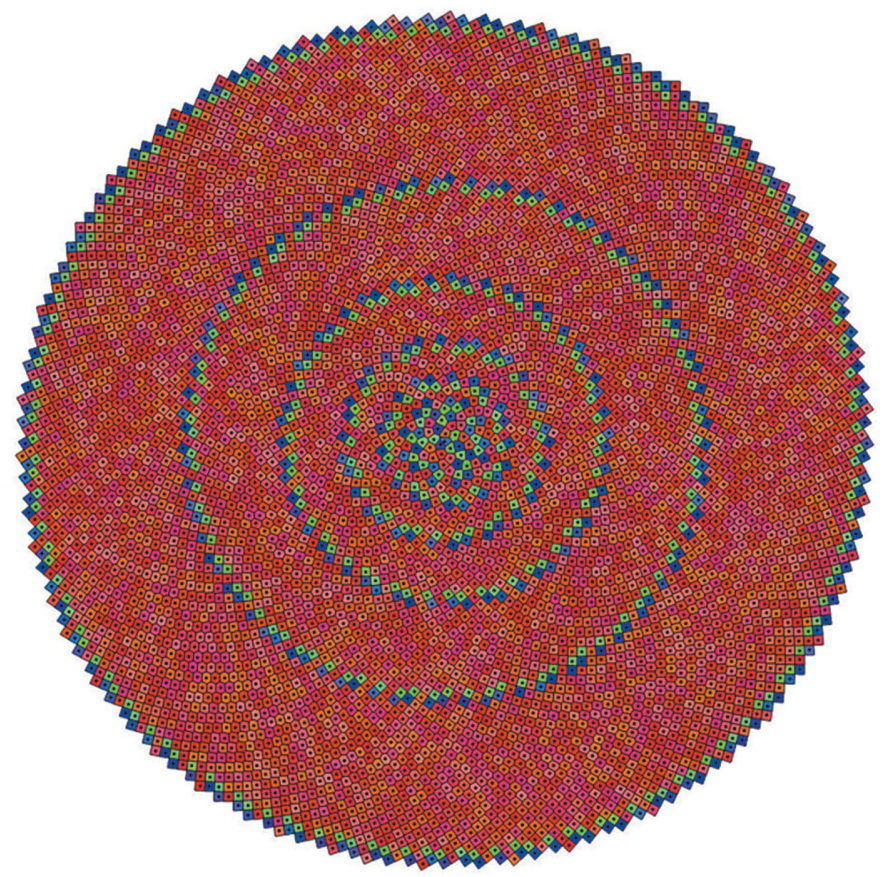

Figure 3

Voronoi decomposition of a 6200 -site model.

proposed. Those propositions are, however, difficult to reconcile with a twisted organization of the fibres, and not compatible with the propagation of any long-range lateral order and the roundness of their normal section, as such orders should lead to facetted sections. As some electron micrographs also suggest a spiral assembly, we recently examined a model in which the sections of the triple helices would be organized according to a phyllotactic pattern (Charvolin \& Sadoc, 2011). Even if no definitive answer can be given, the main features of the X-ray diffraction patterns modelled using phyllotaxis are compatible with experimental results, and we are investigating this problem.

\section{Phyllotaxis}

\subsection{The generative Fermat spiral}

Sites, the points at the centre of physical objects, lie on a spiral (Fig. 1) defined by the equations relating Cartesian coordinates $x, y$ to polar coordinates $\rho(s)=a(s)^{1 / 2}$ and $\theta(s)=2 \pi \lambda s:$

$$
\begin{aligned}
& x(\rho, \theta)=a(s)^{1 / 2} \cos (2 \pi \lambda s), \\
& y(\rho, \theta)=a(s)^{1 / 2} \sin (2 \pi \lambda s),
\end{aligned}
$$

where $a$ is a parameter defining the metric scale, $\lambda$ an important parameter which will be discussed below and $s$ the parametrization of the curve. Sites indexed by integers $s$ are placed on the curve, so that the azimuth between two successive sites varies by $2 \pi \lambda$. The important result of the mathematical studies is that a homogeneous distribution of sites is only obtained with $\lambda=1 / \tau$, the inverse of the golden ratio $\tau=\left(1+5^{1 / 2}\right) / 2$, or $\lambda=1 / \tau^{2}$. The golden ratio is defined 
by $1 / \tau^{2}-1 / \tau-1=0$ so that this second value gives the same result as the two corresponding angles have the same absolute value modulo $2 \pi$ for integer $s$.

Two characteristic properties of this generative spiral are important in order to have the best coverage. First, the added area with any new site must be a constant, for any $\lambda$ : this is the result of $\rho(s) \propto s^{1 / 2}$, but this choice is not sufficient to impose a local isotropy of the distribution of sites. It is the second property of the generative spiral that gives the best local isotropy of the area associated with the best uniform density: the choice of $\lambda=1 / \tau$. With a rational $\lambda$, sites are aligned on straight radial spokes and with irrational $\lambda$ different from $1 / \tau$ (or of a noble number) they form a few long spiralling spokes, so in these two cases sites are gathered on lines (Ridley, 1982; Rivier et al., 1984). Uniformity is indeed associated with the property of 'noble number' to which $\tau$ and $\tau^{2}$ belong, to have only 1 in the tail of their continued fraction expansion. Then these numbers are approximated by successive truncations of their continuous fraction expansion which converge smoothly (Adler, 1998). This point is discussed in Rivier (1988), which defines the best uniformity by the property of having shape and area of Voronoi cells as independent of $s$ as possible, a context-free inflatable structure; see also the so-called 'shape invariance' in Rothen \& Koch (1989a).

We present here structures in which sites define local domains of the same area, but there are also examples where the area changes with the radial position, as in a daisy where external florets are larger than those of the core. These organizations are obtained with other kinds of spiral equations, for instance logarithmic spiral (Rothen \& Koch, 1989a). Nevertheless, conformal transformations but also shearing allow us to explore these different examples of phyllotaxis. This is discussed at the end of this paper.

\subsection{Voronoi tiling}

The geometry of a set of sites can be revealed very well using a Voronoi decomposition of the space (Figs. 2 and 3). In two dimensions, each site is surrounded by a polygonal cell which is the locus of all the points of the plane closer to the considered site than to all other sites. In particular, the Voronoi decomposition strictly defines neighbours of a site whose cell shares an edge with the cell of the considered neighbouring site. Building a Voronoi decomposition for a finite set of sites, we face the difficulty of defining neighbours close to the border of the set. We have solved this by doing the decomposition for $N$ sites embedded in a slightly larger set. With the generic hypothesis of a coordination number $c=3$ for Voronoi cell vertices, topological constraints are imposed by the Euler relation $F-E+V=\chi$, with the Euler-Poincaré characteristics $\chi=0$ for the infinite plane or for any closed surface without Gaussian curvature, like a torus. These constraints are such that the average number of edges of Voronoi cells is strictly six. But a phyllotactic pattern is a finite set with a circular boundary. For a compact finite part of the plane, the Euler relation $V-E+F=\chi$ with the EulerPoincaré characteristic $\chi=1$ has to be considered.
Table 1

Table of cell types in the successive rings or annuli.

Neighbour separations $\delta s$ are Fibonacci numbers, all the same for a given ring, except at the beginning. A label $u$ for large rings corresponds to the Fibonacci number $f_{u}$ giving the medium separation $\left[f_{u}\right.$ in the set of $\left(f_{u-1}, f_{u}, f_{u+1}\right)$ positive separations]. Rings corresponding to defects are marked with $\|$.

\begin{tabular}{|c|c|c|c|c|c|}
\hline \multirow[b]{2}{*}{$u$} & \multirow[b]{2}{*}{ Cell type } & \multirow{2}{*}{$\begin{array}{l}\text { Number } \\
\text { of cells }\end{array}$} & \multicolumn{2}{|l|}{$s$} & \multirow[b]{2}{*}{ Neighbour separations $\delta s$} \\
\hline & & & From & To & \\
\hline & Pentagon & 2 & 0 & 1 & $1,2,3,4,5$ or $-1,2,3,5,8$ \\
\hline & Hexagon & 1 & 2 & 2 & $-2,2,3,5,8,13$ \\
\hline & Heptagon & 3 & 3 & 5 & $\begin{array}{c}(-3,-2,2) \text { or }(-4,-3,-2) \text { or } \\
(-5,-3,-2), 3,5,8,13\end{array}$ \\
\hline & Hexagon & 1 & 6 & 6 & $-5,-3,3,5,8,13$ \\
\hline & Pentagon & 2 & 7 & 8 & $-5,-3,5,8,13$ \\
\hline & Hexagon & 1 & 9 & 9 & $-8,-5,-3,5,8,13$ \\
\hline$\|$ & Hexagon & 5 & 10 & 14 & $-8,-5,5,8,13,21$ \\
\hline$\|$ & Heptagon & 3 & 15 & 17 & $-13,-8,-5,5,8,13,21$ \\
\hline$\|$ & Hexagon & 5 & 18 & 22 & $-13,-8,-5,8,13,21$ \\
\hline$\|$ & Pentagon & 8 & 23 & 30 & $-13,-8,8,13,21$ \\
\hline 7 & Hexagon & 2 & 31 & 32 & $-21,-13,-8,8,13,21$ \\
\hline$\|$ & Heptagon & 13 & 33 & 45 & $-21,-13,-8,8,13,21,34$ \\
\hline$\|$ & Hexagon & 8 & 46 & 53 & $-21,-13,-8,13,21,34$ \\
\hline$\|$ & Pentagon & 13 & 54 & 66 & $-21,-13,13,21,34$ \\
\hline 8 & Hexagon & 34 & 67 & 100 & $-34,-21,-13,13,21,34$ \\
\hline$\|$ & Heptagon & 21 & 101 & 121 & $-34,-21,-13,13,21,34,55$ \\
\hline$\|$ & Hexagon & 13 & 122 & 134 & $-34,-21,-13,21,34,55$ \\
\hline$\|$ & Pentagon & 21 & 135 & 155 & $-34,-21,21,34,55$ \\
\hline 9 & Hexagon & 134 & 156 & 289 & $-55,-34,-21,21,34,55$ \\
\hline$\|$ & Heptagon & 34 & 290 & 323 & $-55,-34,-21,21,34,55,89$ \\
\hline$\|$ & Hexagon & 21 & 324 & 344 & $-55,-34,-21,34,55,89$ \\
\hline$\|$ & Pentagon & 34 & 345 & 378 & $-55,-34,34,55,89$ \\
\hline 10 & Hexagon & 422 & 379 & 800 & $-89,-55,-34,34,55,89$ \\
\hline$\|$ & Heptagon & 55 & 801 & 855 & $-89,-55,-34,34,55,89,144$ \\
\hline$\|$ & Hexagon & 34 & 856 & 889 & $-89,-55,-34,55,89,144$ \\
\hline$\|$ & Pentagon & 55 & 890 & 944 & $-89,-55,55,89,144$ \\
\hline 11 & Hexagon & 1221 & 945 & 2165 & $-144,-89,-55,55,89,144$ \\
\hline$\|$ & Heptagon & 89 & 2166 & 2254 & $-144,-89,-55,55,89,144,233$ \\
\hline$\|$ & Hexagon & 55 & 2255 & 2309 & $-144,-89,-55,89,144,233$ \\
\hline$\|$ & Pentagon & 89 & 2310 & 2398 & $-144,-89,89,144,233$ \\
\hline 12 & Hexagon & 3384 & 2399 & 5782 & $-233,-144,-89,89,144,233$ \\
\hline$\|$ & Heptagon & 144 & 5783 & 5926 & $-233,-144,-89,89,144,233,377$ \\
\hline$\|$ & Hexagon & 89 & 5927 & 6015 & $-233,-144,-89,144,233,377$ \\
\hline$\|$ & Pentagon & 144 & 6016 & 6159 & $-233,-144,144,233,377$ \\
\hline 13 & Hexagon & 9167 & 6160 & 15326 & $-377,-233,-144,144,233,377$ \\
\hline
\end{tabular}

In phyllotaxis there are only hexagonal cells, pentagonal cells and heptagonal cells. Thus, a tiled plane surface enclosed inside a circle in the limit of an infinite radius must have the same number of pentagonal and heptagonal cells. It appears that the three kinds of cells are organized in blocks. Outside a core, there are grains of hexagonal cells which are concentric circular rings, bounded and separated by circular grain boundaries $\left(f_{u-1}, f_{u-2}, f_{u-1}\right)$ made of $f_{u-1}$ heptagonal cells (inner), $f_{u-2}$ hexagonal cells (middle) and $f_{u-1}$ pentagonal cells (outer). The $f_{u}$ are Fibonacci numbers defined by the relation $f_{u}=f_{u-1}+f_{u-2}$ with $f_{1}=1$ and $f_{2}=1$, that is the sequence $1,1,2,3,5,8,13,21,34,55,89,144, \ldots$. These grain boundaries serve as natural boundaries for our optimal packing problem. Outwards packing begins with the first complete grain boundary $(13,8,13)$ with 13 heptagons, 8 hexagons and 13 pentagons. The core disc is bounded by the 8 pentagons of the (first) incomplete grain boundary $(3,5,8)$. It has 3 heptagons instead of the full 8 . The additional pentagons (nearly) fulfil the topological requirement that a tiled disc should have a topological charge of 6 (i.e. 6 additional 


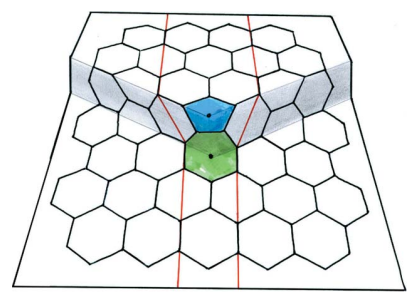

Figure 4

A dislocation in an hexagonal crystal. The figure is drawn with regular polygons, so leading to the appearance of a step. In two dimensions the structure is flattened by projection on a plane.

pentagons, a sphere has a topological charge of 12) (Rivier, 1992; Rivier et al., 2005).

To recapitulate, the hexagons are either in crystalline large grains (in a topological sense) or form narrow rings between heptagonal and pentagonal rings. The numbers of hexagons in the large rings are ...34, 134, 422, 1221, 3384, ... as shown in Table 1.

\section{Rings and defects}

\subsection{Grain boundaries and defects}

In a disordered crystal there are two kinds of disorder with respect to a perfect crystal. The first is metric disorder such that relations of neighbourhood are conserved but the length changes between neighbours; the second is topological disorder, which implies defects like dislocations and disclinations breaking these relations locally. The decomposition of the phyllotactic pattern into rings gives good examples of these two kinds of disorder (Figs. 2 and 3). The large rings of hexagonal cells have metric distortions but without defect. They can be seen as a ribbon cut into a perfect hexagonal crystal and then flattened onto a flat circular ring with its two ends glued, an operation that introduces a change of lengths (on a cylinder, it would be possible to wrap it without distortions). With reference to a perfect hexagonal two-dimensional crystal, pentagons and heptagons are disclinations of opposite weights, but a dipole of opposite disclinations is a dislocation, as shown in Fig. 4. In the Voronoi decomposition, pentagons and heptagons form dipoles which are dislocations in the structure. Several dislocations gathered along a line define a grain boundary between disoriented grains. Hexagonal cells which are between two dipoles inside the dipole ring control the distance between dislocations, and then the disorientation between the two separated large hexagonal rings which are called crystalline grains even if they have metric disorder but without topological disorder. Dislocations are an important factor to keep the best homogeneity of the structure. They are defects relative to the perfect hexagonal crystal, but are intrinsic constituents of the phyllotactic structure.

\subsection{Parastichies}

Parastichy is a botanical term used to describe spiral lines observed on some plants such as composed flowers, cactuses and pine cones. Looking at Figs. 2 and 3 such lines appear, even if they are not easy to follow all the way along. Some segments of parastichies appear in Figs. 5 and 6. The purpose is now to describe them precisely. Points $s$ and $s+1$ placed on the generative spiral are separated by long distances (at least for not too small $s$ ). For visible parastichies we are concerned with points which are neighbours, as defined by Voronoi cells, and search for the separation $\delta s$ between two such points labelled $s$ and $s+\delta s$. Two points with polar coordinates $(\rho, \theta)$ are close if their $\rho$ and $\theta$ are close. So $a(s)^{1 / 2}$ must be close to $a(s+\delta s)^{1 / 2}$, implying a small $\delta s /(2 s)$. The two azimuthal separations must follow $2 \pi \lambda s-2 \pi \lambda(s+\delta s) \simeq 0$ modulo $2 \pi$. It is impossible to have in this equation a strict equality to zero because $\lambda$, the inverse of the golden ratio, is an irrational number, but it can be approximated by a rational number $\lambda^{\prime}=f_{u-1} / f_{u}$, the ratio of two consecutive Fibonacci numbers. The equation for the azimuthal separation, with this approximation, reduces to $2 \pi \lambda^{\prime} s-2 \pi \lambda^{\prime}(s+\delta s)=0$, which is solved exactly by $2 \pi f_{u-1}=0$ modulo $2 \pi$ for $\delta s=f_{u}$. The approximation for $\lambda$ is better for large $u$, but as $\delta s / s$ must be small, first neighbours correspond to the best compromise between radial and azimuthal contribution.

Inside a ring of Voronoi cells of the same type, all neighbours are separated using the same set of Fibonacci numbers

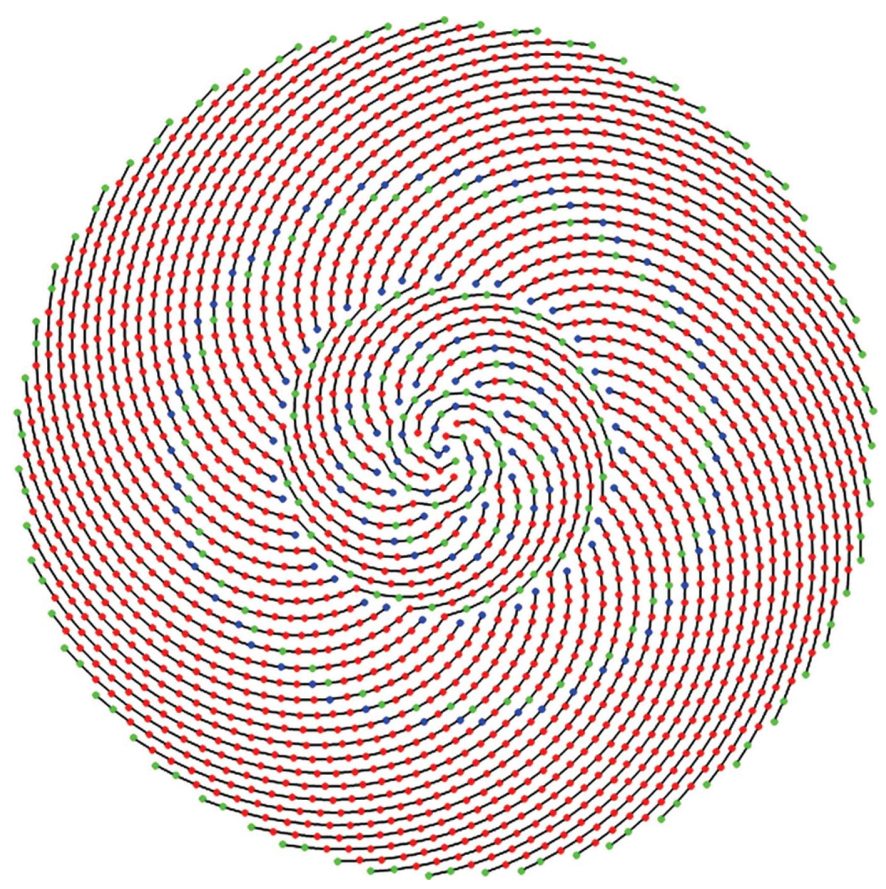

Figure 5

Family of lines formed with parastichy segments, all rotating with the same orientation, corresponding to the smallest separation $\delta s$ given by a Fibonacci number $f_{u}$ with an even $u$ (for conservation of the rotation orientation). The colour code is the same as in Figs. 2 and 3: red for six neighbours, blue for five, green for seven. A new line originates when a new Fibonacci number, with these properties (the smallest with even $u$ ), appears in the list of separations with neighbours. Except for very small $s$, this happens on sites with pentagonal cells. These sites are gathered in one of two rings of pentagonal cells, such that in a heptagon-pentagon dipole the new Fibonacci number is not in the list of separations for the site with the heptagonal cell, but the line is oriented as the dipole. All these lines play the role of reticular lines found in two-dimensional crystals and clearly show the dislocations as an insertion of an extra line. 


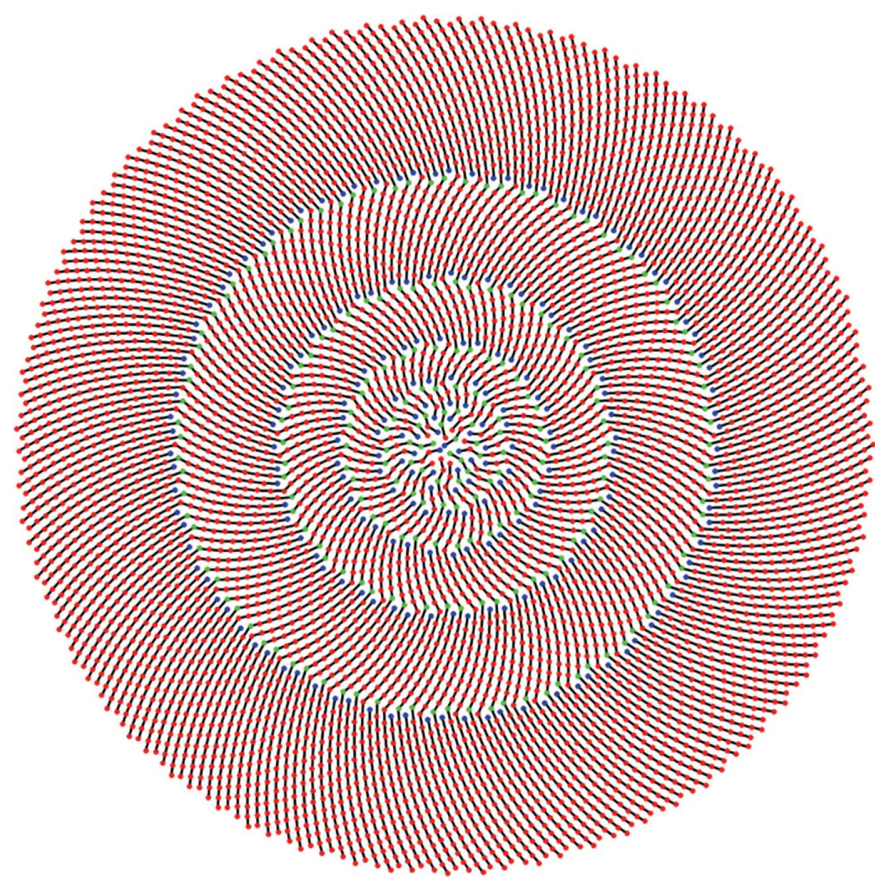

Figure 6

Family of lines formed with parastichy segments, corresponding to the largest separation $\delta s$ given by a Fibonacci number $f_{u}$. Except for very small $s$, new lines originate on sites with pentagonal cells. The number of cells on the border is the number of these parastichies cut by it.

(except for $s<7$ ). For instance, for the ring of hexagonal cells between $s=67$ and $s=100$ all six neighbours are at $s+\delta s$ with $\delta s$ in the set $\{-34,-21,-13,13,21,34\}$ of Fibonacci number $f_{9}=34, f_{8}=21, f_{7}=13$ (Table 1 ).

Passing through a site of large hexagonal rings there are three parastichies defined by three consecutive Fibonacci numbers $f_{u-1}, f_{u}, f_{u+1}$ corresponding to three positive $\delta s$ separations. It is convenient to label large hexagonal rings by $u$ such that $f_{u}$ correspond to the medium separation in this ring. So in the example with $67 \leq s \leq 100$ the label is $u=8$ corresponding to the Fibonacci number $f_{8}=21$ in the set $(13,21,34)$ (see Table 1).

3.2.1. Equation of parastichies. In a grain three visible parastichies are defined by three Fibonacci numbers $f_{u-1}, f_{u}, f_{u+1}$, but now we extend the notion of parastichies to a whole family of curves joining sites separated by any Fibonacci number. So, a parastichy is defined by a given Fibonacci number $f_{u}$. We show that the parastichies equation has the form

$$
\begin{aligned}
& x(\rho, \theta)=a(s)^{1 / 2} \cos \left[\theta_{n}+(-1)^{u+1} 2 \pi \lambda^{u} s / f_{u}\right], \\
& y(\rho, \theta)=a(s)^{1 / 2} \sin \left[\theta_{n}+(-1)^{u+1} 2 \pi \lambda^{u} s / f_{u}\right] .
\end{aligned}
$$

The phase shift $\theta_{n}$ is adjusted in order to have the curve going through the site $s$, so $n$ numbers all parastichies of the same family. Here we consider $s$ as a continuous variable, in order to define a curve, but we assume that the curve passes through sites for integer $s$ values. Consider the two points $s$ and $s+f_{u}$ lying on a parastichy and on the generative spiral. On these curves their azimuthal separation is $2 \pi \lambda f_{u}$. This number, which is much greater than $2 \pi$, can be reduced, modulo $2 \pi$, to a small number between $-\pi$ and $\pi$ given by $2 \pi\left(f_{u} \lambda-f_{u-1}\right)$ because $\lambda \simeq \lambda^{\prime}=f_{u-1} / f_{u}$. The ratio $\lambda^{u}$ appears owing to a property of power of the golden ratio: $\tau^{u}=f_{u} \tau+f_{u-1}$; if $\lambda$ is the inverse of the golden ratio it follows ${ }^{1} \lambda^{u}=(-1)^{u+1}\left(f_{u} \lambda-f_{u-1}\right)$. Introducing the expression for $\lambda^{u}$, this azimuthal separation for $s$ varying from $s$ to $s+f_{u}$ is $(-1)^{u+1} 2 \pi \lambda^{u}$. Consequently the Fermat spiral $\left\{a(s)^{1 / 2} \cos \left[(-1)^{u+1} 2 \pi \lambda^{u} s / f_{u}+\theta_{n}\right]\right.$, $\left.a(s)^{1 / 2} \sin \left[(-1)^{u+1} 2 \pi \lambda^{u} s / f_{u}+\theta_{n}\right]\right\}$ which rotates slowly compared to the generative spiral goes through the two points (with a good choice of the constant $\theta_{n}$ ) when $s$ varies continuously. Notice that the scaling factor $a$ is the same for all spirals including the generative spiral. A factor $(-1)^{u+1}$ changes the orientation of the spirals from even $u$ to odd $u$. This can also be put in relation with the fact that the approximation $\lambda \simeq f_{u-1} / f_{u}$ is an overestimation for odd $u$ and an underestimation for even $u$.

3.2.2. A generalization of parastichies: reticular lines of the phyllotaxis. In botany, parastichies are visible spirals of the structure and so are those joining close neighbour sites. In hexagonal rings there are three such lines characterized by three consecutive Fibonacci numbers; they are clearly visible when their characteristic Fibonacci number appears in Table 1. Nevertheless, for large $s$ even if they are less visible they always exist as continuous spirals joining sites not necessarily first neighbours, but second, third ... neighbours. So, to each possible Fibonacci number $f_{u}$ corresponds a family of $f_{u}$ identical spirals joining sites with a separation $\delta s=f_{u}$. There are $f_{u}$ in the family, because if one goes through the site $s$ and also through the site $s+f_{u}$, there is one through the site $s+1$ which is different, and one through $s+2$ and so on up to $s+f_{u}-1$.

The first example is the family corresponding to $f_{1}=1$ with only one member, the generative spiral joining site $s$ and $s+1$. The next example is given by $f_{2}=1$, also joining sites $s$ and $s+1$. These are the first two families with a single spiral in each. In fact these two different spirals correspond to the two possible choices of $\lambda$ given by $1 / \tau$ and $1 / \tau^{2}$ giving the same structure with two spirals with reversed rotation orientations. ${ }^{2}$

\subsection{Euler relation}

As already mentioned above, the Euler relation $V-E+F=\chi$ imposes the average number of edges per Voronoi cell to be six on the infinite plane for which $\chi=0$. However, for a compact finite part of the plane, such as the circular domain of a phyllotactic pattern, $\chi=1$ so that the numbers of cells with five or seven edges are not equal. We introduce the number of faces $F=F_{5}+F_{6}+F_{7}, F_{p}$ being

\footnotetext{
${ }^{1}$ These two relations can be shown easily by induction considering that they are true, then calculating $\tau^{u+1}$ or $\lambda^{u+1}$ using $\tau^{2}=1+\tau$ or $\lambda^{2}=1-\lambda$.

${ }^{2}$ It is very usual in the literature to use $\lambda=1 / \tau^{2}$ to describe phyllotaxis. This choice corresponds to the smaller divergence angle. The other choice, done here, is formally simpler and strongly supported by this property: spirals are defined by the rank $u$ of the Fibonacci number; their speeds of rotation around the origin decrease with $u$ and we choose for a generative spiral the first one given by $u=1$.
} 
the number of faces with $p$ edges, and the number of edges $E=\left(5 F_{5}+6 F_{6}+7 F_{7}\right) / 2+\left(m_{2}+m_{3}\right) / 2$ or in order to eliminate $V, E=3\left(V-m_{2}-m_{3}\right) / 2+\left(2 m_{2}+3 m_{3}\right) / 2$. The numbers $m_{2}$ and $m_{3}$ are the number of vertices of the cells on the border with coordinations $c_{r}=2$ or 3 . This gives $\left(m_{2}-m_{3}\right)+\left(F_{5}-F_{7}\right)=6$. Two particular solutions of this equation are interesting. First, following what happens on the infinite plane, suppose that $F_{5}=F_{7}$ so there is $m_{2}=6+m_{3}$, an excess of six sites with coordination 2 on the border. A simple example is obtained with a finite piece of a hexagonal tiling cut along the edges of hexagonal cells (so with $F_{5}=F_{7}=0$ ). Then this structure has an irregular boundary depending on the choice of hexagons kept inside it respecting $m_{2}=6+m_{3}$ for the $m_{2}$ sites of the boundary belonging to only one hexagon, and the $m_{3}$ sites belonging to two hexagons. The other particular solution corresponds to the phyllotaxis: the boundary is close to a circle with $m_{2}=m_{3}$, an alternative equipartition of coordination 2 and 3 on the border. Notice that in a perfect hexagonal tiling cut along the edges in order to divide it into two pieces with sites on the cut alternately on one or two hexagons $\left(c_{r}=2\right.$ or 3$)$, the cutting line oscillates around a straight line, so we can consider such a border as some kind of 'topological geodesic' (Rivier et al., 2005). In this case, solving the equation gives $F_{5}-F_{7}=6$ corresponding to an excess of six pentagons in the core of the structure. Evidently a circular border with these properties $\left(m_{2}=m_{3}\right.$ forcing $F_{5}-F_{7}=6$ ) must be inside a large hexagonal domain and not inside rings of defects: otherwise there would be an excess of heptagon. A consequence of $m_{2}=m_{3}$ on the border is also that the number of cells on the border (having outside edges) does not vary continuously with the total number of sites in the phyllotactic pattern: this number remains constant in large hexagonal rings, but otherwise changes rapidly when the boundary enters inside a grain boundary. This number is equal to the number of parastichies corresponding to the highest separation between neighbours, so if the border is inside a non-defective domain it is equal to Fibonacci numbers.

\subsection{Rings and defects: a relationship with crystals}

Exactly like for reticular lines in a two-dimensional crystal, all sites are on a member of each family and lines of the same family are in some sense parallel lines: in crystals they are equivalent by translation, here they are equivalent by rotation. In two-dimensional crystals, a reticular family is characterized by two Miller indices but the family of a spiral is just characterized by a Fibonacci number. To recapitulate, the number of spirals of the same family $f_{u}$ is the Fibonacci number $f_{u}$. As the golden ratio $\tau$ is approximated by the ratio of successive Fibonacci numbers converging towards it alternately up and down, this results in an alternate rotation orientation for the spirals depending on the parity of $u$ in $f_{u}$.

There are relationships between crystals with defects and phyllotactic patterns, but the next section describes relationships to quasicrystals.

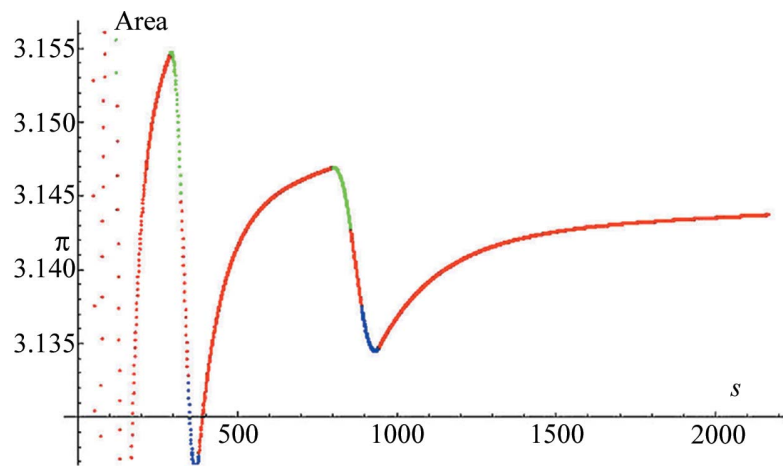

Figure 7

Variation of the area of Voronoi cells for points between $s=34$ and 2166 . Colours correspond to the type of Voronoi cells. The area is close to the mean value $\pi$ with $a=1$, rapid variations appear in rings of defects. Nevertheless these strong variations decrease as the area tends towards $\pi$ for large $s$.

\section{Metric properties}

\subsection{Packing density}

As the circle of radius $\rho$ and area $\pi \rho^{2}=\pi a^{2} s$ contains $s$ points, the area added per point has the value $\pi a^{2}$. The area of Voronoi cells oscillates close to this value for small $s$ and then converges towards it (Fig. 7).

\subsection{Influence of site distances on shapes of Voronoi cells}

In the Voronoi decomposition of a phyllotactic pattern there are only hexagons, pentagons and heptagons; nevertheless, looking rapidly at Fig. 3, some squares seem to appear, mainly in rings related to dislocations. In fact these are not squares, but Voronoi cells which could be described as a square with one corner slightly truncated (for a pentagon), or with two truncated corners (for a hexagon), or three truncated corners (for a heptagon). This is related to the fact that the two parastichies corresponding to the first two Fibonacci separations are close to being orthogonal in grain boundaries. Oppositely, in large rings of hexagonal cells, the core of the ring contains less irregular hexagonal cells.

Fig. 8 shows how Delaunay triangles are organized near the grain boundaries. ${ }^{3}$ In these regions corresponding Voronoi cells are close to being square, but with a corner slightly truncated. Effectively Voronoi cell vertices are centres of circumscribed circles of Delaunay triangles, so if two such triangles look like a square divided by a diagonal, the two centres are close to the diagonal leading to a small edge of the Voronoi cell. If two corners are truncated the cell is a hexagon. In large hexagonal rings, the Voronoi cells are a rhombus with two opposite truncated corners, so the Voronoi cells are close to regular hexagons. In the narrow hexagonal rings, inside grain boundaries, the cells look like squares with two close truncated corners, so are very distorted hexagons.

\footnotetext{
${ }^{3}$ Delaunay triangulation is such that sites are associated to cover the surface by triangles. It is related to Voronoi decomposition, because the vertices of Voronoi cells are the centres of the triangles, Delaunay and Voronoi decompositions are dual.
} 


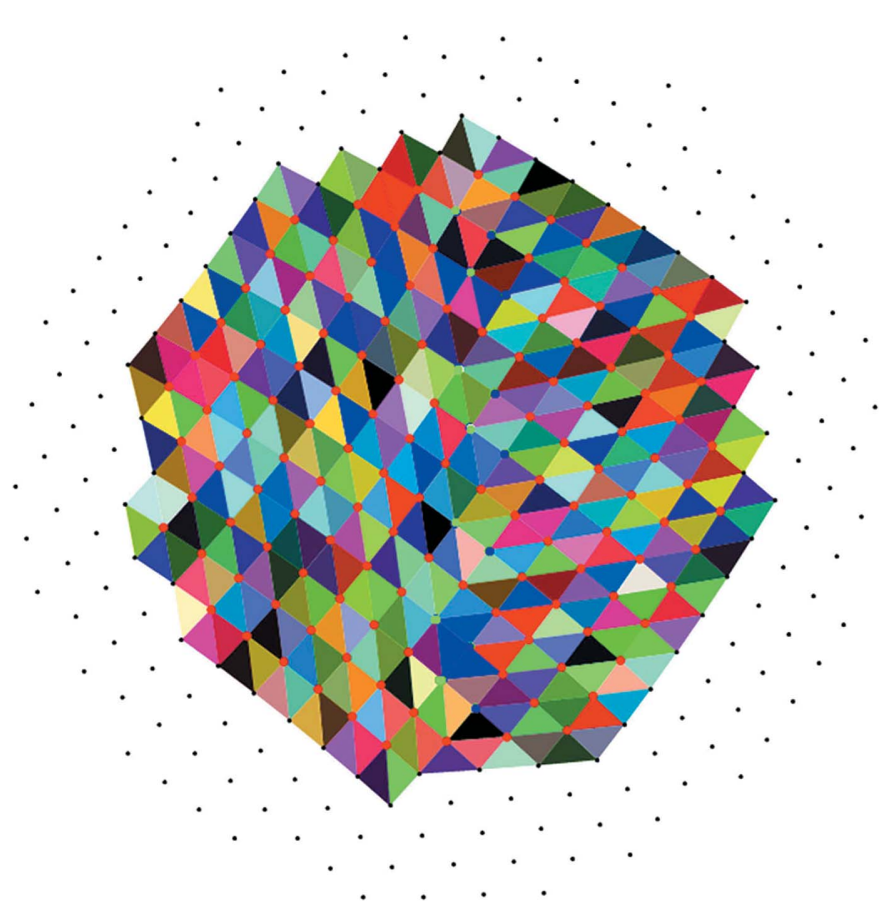

Figure 8

Delaunay triangulation in a small region around the point $s=15326$, which is the last point in a wide six-neighbours ring. The centre of the phyllotactic disc is far away on the left. Red points are common to six triangles, blue points to five triangles and green points to seven triangles. Often it seems that two triangles form a square, but in fact in this sheared square the diagonal which divides it into triangles is slightly shorter than the other one. There are two domains (grains) distinguished by the orientations of the diagonals. In these domains all points are common to six triangles. Between the two domains the defect ring (grain boundary) shows up as a flip of the diagonal orientations, leading to seven, five or sometimes six triangles around a point. Going away from the grain boundary, the triangles become more and more regular.

Even if Delaunay and Voronoi decompositions give exactly the same information, some properties are more easily observed with one or the other method. For instance, angles between parastichies are very clear in Fig. 8 because the edges of triangles are along parastichies. It is clear in this region of defects that two families of parastichies are close to being orthogonal with a third family making a $\pi / 4$ angle with the others.

\subsection{Distances between first neighbours}

The behaviour of distances between first-neighbour sites is shown in Fig. 9. In domains where Voronoi cells are slightly truncated squares, their area is approximatively $\pi a^{2}$, so that the distance between points along a square edge is $\pi^{1 / 2} \simeq 1.772$ (with $a=1$ ) and along a diagonal is $(2 \pi)^{1 / 2} \simeq 2.506$. When Voronoi cells are more clearly hexagons two distances are close to $\left(3 \pi / 5^{1 / 2}\right)^{1 / 2} \simeq 2.053$. The behaviour of the distance between the two points defined by $s$ and $s+f_{u}$ as a function of $s$ is obtained using a relation given by Yeatts (1997):

$$
d_{u}(s)=f_{u}\left[\frac{1}{4 s}+\frac{s\left(-2 \pi f_{u-1}+2 \pi \tau^{-1} f_{u}\right)^{2}}{f_{u}^{2}}\right]^{1 / 2} .
$$

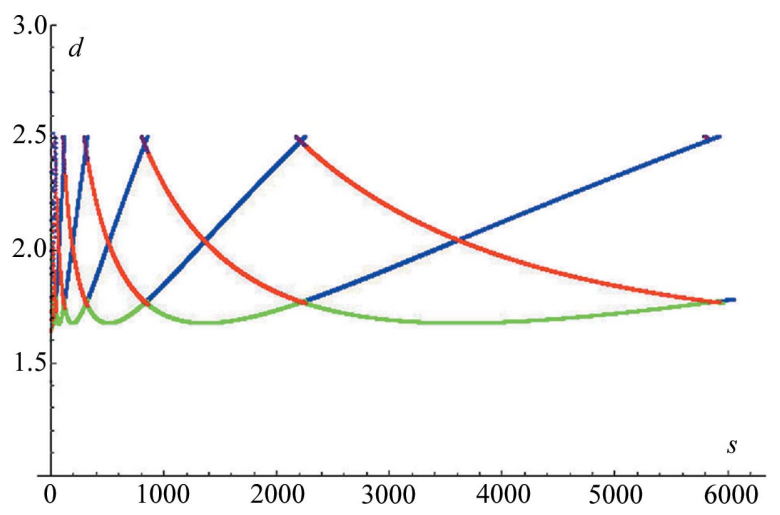

Figure 9

First-neighbour distances between sites $s$ and $s+\delta s$. Blue for the interval $\delta s$ equal to the smaller positive Fibonacci number in the list of Table 1; green for the next interval; red for the third positive interval; and purple for the last one (occurring only if the Voronoi cell is a heptagon). Thus green, blue and red correspond to distance along the three visible parastichies. Each continuous curve corresponds to a given Fibonacci number that appears in different annuli. For instance, $f_{11}=89$ appears between $s=290$ and 5926 leading to a continuous curve which is successively purple, red, green and blue. Each lower crossing of two curves corresponds to a grain boundary, the upper crossing is in the middle of a hexagonal ring. The scaling parameter in equation (1) is $a=1$, so the distances are approximately in the range $[1.676,2.506]$ with a mean value 1.903 .

This relation fits perfectly the numerical values given in Fig. 9. It can be checked that minimal values

$$
(2 \pi)^{1 / 2} f_{u}\left(\left|\frac{1}{\tau}-\frac{f_{u-1}}{f_{u}}\right|\right)^{1 / 2}
$$

are all close to and converging towards $\left(2 \pi / 5^{1 / 2}\right)^{1 / 2} \simeq 1.67$.

All distances are in the range $\left[\left(2 \pi / 5^{1 / 2}\right)^{1 / 2},(2 \pi)^{1 / 2}\right]$, whatever $s$ and $u$ will be. This property, specific to the choice of $\lambda=1 / \tau$, is very important to ensure the best packing homogeneity. ${ }^{4}$

\section{Grain boundaries}

Table 1 shows the lower and upper bounds of domains containing the same kind of Voronoi cells. These values are obtained from numerical observations of a phyllotaxis. Nevertheless it would be interesting to have an analytic relation giving these bounds. Clearly, by having the lower bound for heptagonal or pentagonal rings the upper bound is obtained by adding a Fibonacci number minus one. This is also true for a narrow hexagonal domain, in the same region, using the previous Fibonacci number. But there is no clear relation for wide hexagonal rings. We introduce a semi-empiric relation which has been tested up to $s=280783$.

As in a grain boundary Voronoi cells are close to being squares, we suppose that sites are with a good approximation on a square lattice with parameter $d=\pi^{1 / 2}$. We set $a=1$ in

\footnotetext{
${ }^{4}$ These limits remain the same in phyllotactic tilings on the sphere and on the hyperbolic plane, providing the same $\pi$ area for each site. This will be discussed in a further article on phyllotaxis in non-Euclidean geometry.
} 


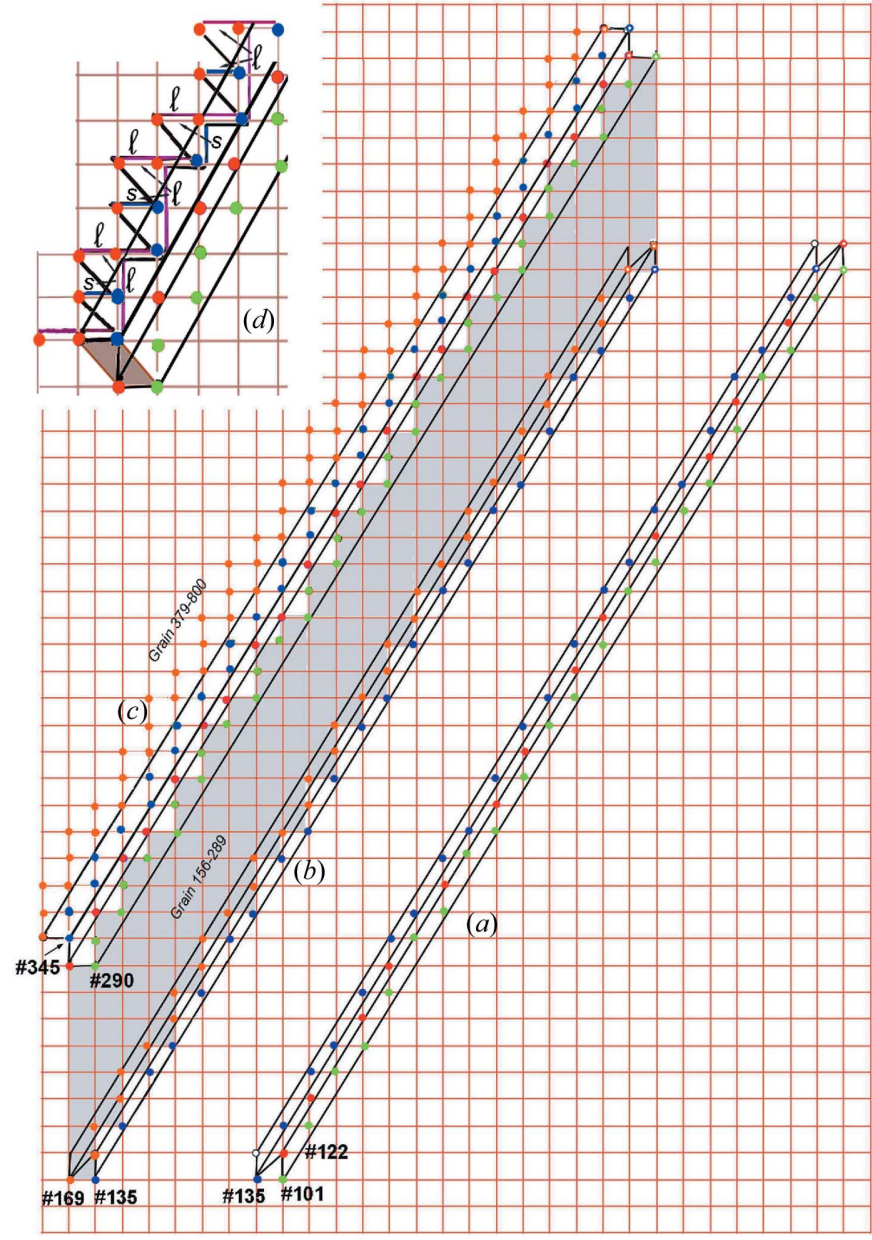

Figure 10

Strip cut in a square lattice $(a)$. This strip is a good approximation of the ring of defects between points $s=101$ and $s=155$ containing 55 points. The colour of the points corresponds to their type of Voronoi cells. Point $s=101$ is the first green point down, then points are numbered, increasing by 21 going up or decreasing by 34 going right. This strip can be divided into three strips of heptagons, hexagons and pentagons. $(b, c)$ Continuation of the grain towards the other boundary on the same square grid $\{(x, y): x-1 \leftrightarrow s+34, y-1 \leftrightarrow s+21\}$ which can be considered as folded on a cylinder with axis perpendicular to the strip. This grain (in grey) is limited by an inner border $(b)$ and an outer border $(c)$. Blue points (pentagons) in $(b)$ are those of $(a)$, orange points belong to the large grain. The grain is bordered in $(b)$ by a Fibonacci chain defined by the lower part of the grey domain. There are 21 steps in it containing 21 blue points and 34 orange points (hexagons of the large grain). The other border of the grain [in (c)] is geometrically identical, but it contains 34 green points (heptagons of the next ring of defects) and 21 red points (hexagons of this ring of defects). This next ring of defects from $s=290$ to $s=378$ contains 89 points. The beginning of this ring is expanded in (d), with two Fibonacci chains, one which closes the grain from $s=156$ to $s=289$ and the other which begins the next grain, from $s=379$ to $s=800$. The two Fibonacci chains could be coded with long (purple) and short (blue) segments forming a 'staircase'. Steps of the first chain appear with $\pi / 2$ angles, but for the other angles are $\pi / 4$. In fact the strip should be deflated (stretched along by a rational approximant of $\tau$ and compressed across by an approximant of $1 / \tau$, that is a Poisson shear). Introducing progressively the Poisson shear, instead of a part of a cylinder the substrate of the grain and its two boundaries are a part of a surface with a negative constant Gaussian curvature around the same axis. This Poisson shear changes the rhombic cell, darker in $(d)$, into a square. Refer also to Figs. 11 and 12. the following. Fig. 10(a) shows a representation of the grain boundary at $101 \leq s \leq 155$ in which Voronoi cells are 21 heptagons, 13 hexagons and 21 pentagons. It appears straight in this representation, but in the phyllotaxis it is refolded into a ring. The grain boundary appears as a strip of length given by the vector $(21,34)$ in the square lattice and of width given by $(1,-1)$, so that the number of points in the strip is 55 , given by the determinant constructed with the two vectors. This strip is separated into three thinner strips of the same length, but with widths defined by vectors $(0,-1),(1,1)$ and $(0,-1)$, respectively. The first one contains the 21 points with heptagonal cells, the second contains 13 points with hexagonal cells and the third contains 21 points with pentagonal cells. ${ }^{5}$ In the phyllotactic pattern, the 55-point strip is folded into a flat ring that bounds a disc, by identification of the two small sides at the cost of metric distortions.

We estimate the bounds of the strip using two properties: the length of the strip and the number of points in the disc that are enclosed with radius $s^{1 / 2}$. The length is the module of the vector $(21,34)$ which is $L=\left(21^{2}+34^{2}\right)^{1 / 2} \pi^{1 / 2}$ using $d=\pi^{1 / 2}$ as length unit. Folding the strip into a ring, this length is the perimeter of a circle. We can estimate the number of points of the phyllotactic pattern in the disc enclosed by this circle using the definition of the generative spiral. This number is $s_{b}=$ $\left(21^{2}+34^{2}\right) / 4 \pi \simeq 127.085$.

Now we compare this to the known value for the upper and lower bounds in this grain boundary. When the strip is folded into a ring, the internal side of the ring is compressed whereas the external side is expanded, so $s_{b}$ is collimated by the narrow ring of hexagons. The evaluated value $s_{b}$ falls between these bounds $(122,134)$.

From this estimation we deduce a formula which gives the bounds of all hexagonal rings appearing inside defect regions

$\left[\operatorname{round}\left(\frac{3-f_{u-1}}{2}+\frac{f_{u}^{2}+f_{u+1}^{2}}{4 \pi}\right)\right.$, round $\left.\left(\frac{f_{u-1}+1}{2}+\frac{f_{u}^{2}+f_{u+1}^{2}}{4 \pi}\right)\right]$.

It is possible to write this relation in a more compact way using the identity $f_{u}^{2}+f_{u+1}^{2}=f_{2 u+1}$, which can be shown by inductive reasoning on $v$ using $f_{u+v}=f_{v+1} f_{u}+f_{v} f_{u-1}$. Then we have

$$
\left[\operatorname{round}\left(\frac{3-f_{u-1}}{2}+\frac{f_{2 u+1}}{4 \pi}\right) \text {, round }\left(\frac{f_{u-1}+1}{2}+\frac{f_{2 u+1}}{4 \pi}\right)\right] \text {. }
$$

Here $f_{u}$ is the Fibonacci number of order $u$ and the function round takes the integer part of an irrational number. The example of Fig. 10 corresponds to $f_{8}=21$. In this relation the two bounds are such that the number of points in the ring is $f_{u-1}$ as wanted. This formula has been checked to give an exact value (except a shift of one for $u=11$ ) up to the bounds (280174, 280783) obtained with $u=16$. Using the fact that rings involved in defect domains have a number of points given by a Fibonacci number, it is easy to get all other bounds as given in Table 1 .

\footnotetext{
${ }^{\mathbf{5}}$ Notice that this strip is very similar to the construction of approximants of a quasicrystal using the cut-and-projection method. It is one of the factors that relate phyllotaxis and quasicrystals.
} 
There is another way to look at this problem, which is to search $s$ for points where two parastichies are very close to being orthogonal. This relation, which is deduced from the results given by Yeatts (1997), is $s=$ $\left\{\operatorname{abs}\left[\left(f_{u} f_{u+1}\right) /\left(4 \gamma_{u} \gamma_{u+1}\right)\right]\right\}^{1 / 2}$, where $\gamma_{u}$, related to approximants of $\tau$, is $\gamma_{u}=2 \pi\left(\lambda f_{u}-f_{u-1}\right)$. This relation gives real values for $s$ very close to $\left(f_{2 u+1}\right) / 4 \pi$ but clearly different (for instance $s_{b}^{\prime} \simeq 127.067$ compared to $s_{b} \simeq 127.085$ ).

It will be a challenge to find an exact relation giving integer values for the bounds without using the functions 'round', 'floor' or 'ceiling' extracting integers from non-integer numbers.

The ratio between the radii of two successive grain boundaries is proportional to $\left(f_{2 u+1} / f_{2 u-1}\right)^{1 / 2}$, very close to the golden ratio.

\section{Conformal transformations and shearing in phyllotaxis}

In this section we show how the successive grains are sheared relative to a perfect crystalline structure, so that each parastichy is a stack of deformed hexagons, starting from a square at one boundary, through to a more symmetrical hexagon at mid-grain, to a square at the next boundary. See Fig. 9, where the lowest crossings correspond to square cells at the grain boundary with $d=\pi^{1 / 2}$ [the third distance is the diagonal $(2 \pi)^{1 / 2} \simeq 2.507$, the maximal distance]. The upper crossings correspond to hexagons with $2 \pi / 3$ angles (but not perfectly regular) at mid-grain, with a minimal distance given by equation (4).

Phyllotaxis [equation (1)] is an example of a spiral lattice (Rothen et al., 1993), the image $D$ in the complex plane $\mathbf{w}=\rho \exp i \theta$ of a regular lattice $P$ in a domain of the complex plane $\mathbf{z}$ with Cartesian coordinates. In elasticity, $D$ is the actual, deformed state of the material, and $P$ is called the natural state. The mapping is through the function $\mathbf{w}(\mathbf{z})$.

One particular mapping $\mathbf{w}(\mathbf{z})=\mathbf{w}_{0} \exp \left(\mathbf{b}^{*} \cdot \mathbf{z}\right)$ is conformal, i.e. it is analytic, invertible, singularity free (excluding a small domain around the origin of $\mathbf{w}$ ), and thus without any defect (dislocation, disclination or grain boundary). It conserves the angles and is also unsheared (a consequence of the CauchyRiemann relations imposing analyticity) (Rothen et al., 1993).

We consider a square lattice and we define its node positions by complex numbers in its plane. As an example we consider a strip in this lattice, like that shown in Fig. 10 with square vertices selected by it. We have to map it on a ring of a dipole. In this example the strip is defined by a long multiple cell of the square lattice characterized by the two vectors $\mathbf{b}_{2}=(21,34)$ and $\mathbf{b}_{0}=(1,-1)$. The conformal mapping transforms the imaginary axis into a circle, so we have to choose this axis along the $\mathbf{b}_{2}$ vector. As complex numbers are defined using orthogonal real and imaginary axes, we introduce the real axis along the vector $\mathbf{b}_{1}=(34,-21)$. To express the argument of the mapping function we introduce reciprocal vectors of $\mathbf{b}_{1}$ and $\mathbf{b}_{2}$ called $\mathbf{b}_{1}^{*}$ and $\mathbf{b}_{2}^{*}$ and defined by $\mathbf{b}_{i} \cdot \mathbf{b}_{j}^{*}=\delta_{i j}$. Then the mapping function is $\mathbf{w}(\mathbf{z})=$ $\mathbf{w}_{0} \exp \left[2 \pi\left(\mathbf{b}_{1}^{*}+i \mathbf{b}_{2}^{*}\right)\left(x \mathbf{b}_{1}+y \mathbf{b}_{2}\right)\right]$, where $x$ and $y$ are coordi- nates of a point $\mathbf{z}$ expressed in the multiple cell. The mapping function reduces simply to $\mathbf{w}(\mathbf{z})=\mathbf{w}_{0} \exp [2 \pi(x+i y)]$ with the scaling parameter $\mathbf{w}_{0}$.

The square lattice on $P$ includes all points with $s \geq 101$. Note the particular families of reticular lines, (i) $(1,0),(0,-1)$ and $(1,-1)$, that are mapped into parastichies, (ii) $(21,34)$, parallel to the grain boundary, that are mapped into concentric circles, and (iii) $(34,-21)$ that are mapped into radial spokes. The conformal mapping yields a spiral lattice (Rothen \& Koch, 1989a), with all reticular lines mapped into equiangular (or logarithmic) spirals, save $(21,34)$ (concentric circles) and $(34,-21)$ (radial spokes). All angles are conserved in the mapping, notably the two parastichies, images of $(1,0)$ and $(0,-1)$ remain at right angles and keep the same angle with the circles parallel to the grain boundary and with the perpendicular radial spokes. Thus, Fig. 10 is a conformal (literally) representation of the grain boundary, with square-shaped Voronoi cells. However, the density of points $|d w / d z|^{-2}=|b w|^{-2}$ is not uniform and the conformal mapping must be sheared, from the grain boundary outwards, to achieve the required uniformity. Accordingly, the hitherto defect-free phyllotaxis will exhibit other grain boundaries further out. Remarks: $(a)$ the mapping is enacted outwards, as occurs naturally in the growth of phyllotactic structures, younger florets pushing out the older ones and squeezing inbetween at grain boundaries; $(b)$ in the central core of the phyllotaxis, for small $s$ in the range $s<32$, it is difficult to identify precise rules even if the structure corresponds to the smoothest way to accommodate non-singular topological defects (Rivier et al., 2005).

Fig. $10(a)$ is a representation of the lattice $P$. It consists of adjacent, non-overlapping parallel strips of equal thickness generated by the translation $\mathbf{b}_{1}$. Each strip is bounded by two reticular lines parallel to $(21,34)$ and contains 55 points, the diagonal of the unit square goes as the thickness of the strip. The two points are separated by $\delta s=55$. The first strip is the grain boundary itself. Successive strips are mapped conformally by $\mathbf{w}(\mathbf{z})=\mathbf{w}_{0} \exp [2 \pi(x+i y)]$ into concentric rings containing 55 points each, but with thickness increasing exponentially $\rho(s+55)=\rho(s) \exp (55)$, where $\rho=|w|$. In order to have a uniform density of sites in the presented phyllotaxis with $\rho(s)=a(s)^{1 / 2}$, the successive strips in $P$ must be squeezed across and extended through a Poisson shear strain (Fig. 10d).

\section{Relation with quasiperiodic structures}

The way dislocation dipoles are organized along circles is strongly related to one-dimensional quasicrystals (Rivier, 1986). A Fibonacci one-dimensional quasicrystal can be obtained using an inflation-deflation rule iteratively applied to a sequence of long and short segments. This inflation-deflation rule is $L \rightarrow L+S$ and $S \rightarrow L$. Starting simply from a short segment, this specific rule gives a quasicrystal after an infinite number of iterations, or with a given number of iterations, a finite structure with a number of short and long segments given by two successive Fibonacci numbers. Consider dipoles 
along circles in the phyllotaxis. There are isolated dipoles (a heptagon and a pentagon in contact) and a pair of close dipoles. Consider now the rule: change an isolated dipole into a pair of dipoles and change a pair of dipoles into a pair and an isolated dipole. It is the rule we have going from one ring of dipoles to the next ring. So there is an inflation-deflation symmetry associated with radial change relating defects in this structure. This can be checked by counting the number of pairs of dipoles or of isolated dipoles which are successive Fibonacci numbers, on circles of defects. Fig. 10, which is similar to figures used in the presentation of a one-dimensional quasicrystal defined by cut and projection, is related to this property.

\subsection{Successive grain boundaries}

The first complete grain boundary $(13,8,13)$ marks the beginning of a regular, inflatable phyllotaxis, where the circular grain boundaries are words, sequences of letters $L$ and $S$, where $L$ labels a dislocation (oriented dipole 7/5) and $S$ an isolated hexagon in the grain boundary. The sequences are obtained by inflation rules $S \rightarrow L, L \rightarrow L S$. Consider three successive grain boundaries, with sequences $w_{i-2}, w_{i-1}$ and $w_{i}$. Inflation implies that the sequence $w_{i}$ is given by concatenation of the previous two, $w_{i}=w_{i-1} \bullet w_{i-2}$, thus $w_{i}=L S L L S L S L$ is a concatenation of $w_{i-1}=L S L L S$ with $w_{i-2}=L S L$. The word $w_{i}$ has $\left|w_{i}\right|=f_{i}$ letters, $f_{i-1} L$ and $f_{i-2} S$. It starts with $L S$ and ends with $L S$ for $i>3$ with even $f_{i}$, with $S L$ for $i>3$ with odd $f_{i}$. A circular word is also a palindrome. Let $w^{T}$ be the sequence $w$ read backwards (transposed in matrix notation). A palindrome $p=p^{T}$ is a sequence of letters that reads the same either way, e.g. $L S L S L$, 'hannah' or 'eve'. The word $w_{i}$ can also be written as the concatenation of two palindromes $w_{i}=p_{A} \bullet p_{B}$, with $w_{i-1}=p_{A} S L, p_{B}=S L w_{i-2}$ for an even number of letters, and $w_{i-1}=p_{A} L S, p_{B}=L S w_{i-2}$ for an odd number of letters. Thus $\left|p_{A}\right|=f_{i-1}-2$, $\left|p_{B}\right|=f_{i-2}+2$. For inflation to be consistent from one grain boundary to the next, one requires $\left|p_{A}\right|>\left|p_{B}\right|$, so that the shortest complete grain boundary is $(13,8,13)$, a palindrome of 21 letters $L$ and $S$, written on a circle and readable in either sense, since $w=p_{A} \bullet p_{B}=p_{B} \bullet p_{A}$ (by circular invariance) equals $w^{T}=p_{B}^{T} \bullet p_{A}^{T}$.

Thus, $p_{A}^{i}=p_{A}^{i-1} L S p_{A}^{i-2}=p_{A}^{i-2}[S L] p_{A}^{i-3}[L S] p_{A}^{i-2}$ for $i$ even, and $p_{A}^{i}=p_{A}^{i-1} S L p_{A}^{i-2}=p_{A}^{i-2}[L S] p_{A}^{i-3}[S L] p_{A}^{i-2}$ for $i$ odd, with $p_{A}^{3}=1$.

\subsection{Boundary of large hexagonal rings}

A large hexagonal ring lying on the plane is nevertheless topologically equivalent to a perfect hexagonal tiling wrapped around a finite cylinder limited by two parallel circles. With such distortion of the ring, hexagonal cells are perfect regular hexagons and so this geometry can be seen as a multiple crystallographic cell of the hexagonal lattice with two identified opposite sides. The vectors that are the basis of the multiple cell entirely characterize the topology of its related large hexagonal ring. In order to precisely define the bound-

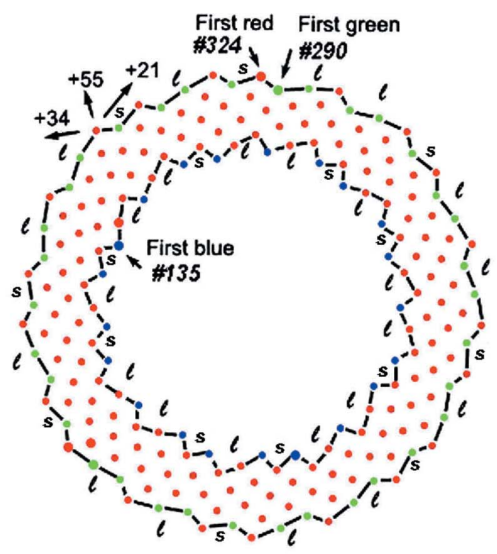

(a)

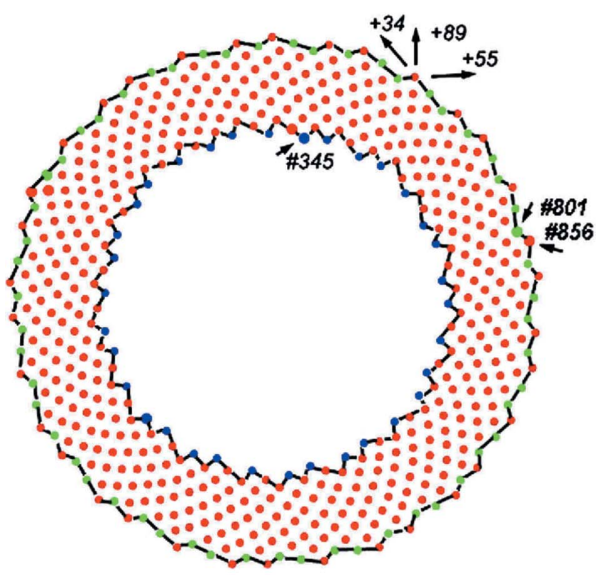

(b)

Figure 11

Two examples of hexagonal rings: (a) with 134 hexagonal sites (red), completed with 21 pentagonal sites (blue) on the inner border and with an outside border containing 34 heptagonal sites (green) and 21 hexagonal sites (red); $(b)$ with 422 hexagonal sites (red), completed with 34 pentagonal sites (blue) on the inner border and with an outer border containing 55 heptagonal sites (green) and 34 hexagonal sites (red). The inner border has blue points inside, with zigzag steps between blue points containing one or two red points. This is labelled $l$ or $s$ in $(a)$. The outer border has red points on the outside with one or two green points between two red points. The inner and outer sequences of $l$ and $s$ are the same, starting at the first 'blue point' or the first 'green point'. Three families of parastichies are clearly visible: they are characterized by three Fibonacci numbers $\left(f_{u-1}, f_{u}, f_{u+1}\right)$ indicating the increase of indices jumping from a point to a neighbouring point following a parastichy. These numbers are indicated on the figure [they correspond to $u=9$ for (a) and $u=10$ for $(b)$ ]. Notice that if we orient the border following parastichies defined by the smaller increase given by $f_{u-1}$, the borders in $(a)$ and $(b)$ are in reverse orientation: this is associated with the parity of $u$.

aries of a ring we will consider in it all pentagonal sites which are just before and all heptagonal sites just after associated with the following narrow hexagonal ring. With this definition of large ring boundaries, the set of all large rings entirely covers continuously the whole structure. They are perfect topological hexagonal structures, each defined by a crystallographic multiple cell, the multiplicity of which is given by the number of points in it. The departure from the hexagonal coordination only results from the junction between successive rings. 
The inner border of a ring is a zigzag line containing pentagonal sites whose number is given by a Fibonacci number $f_{u-1}$ and hexagonal sites whose number is $f_{u}$. Fig. 11 gives two examples of hexagonal rings: one containing 134 hexagons, characterized by the medium neighbour separation $\delta s=f_{9}=34$, the other with 422 hexagons and $\delta s=f_{10}=55$. Consider such a ring as a hexagonal lattice, with points on vertices of regular triangles, wrapped around a cylinder and with the primitive cell defined by two vectors $\mathbf{a}, \mathbf{b}$ defining elementary translations from a point to its first neighbours defined by $f_{u-1}$ and $f_{u}$. Then the ring can be seen as a multiple cell defined by two vectors expressed in the primitive cell by coordinates $\left(f_{u-1},-f_{u}\right)$ and $(x, y)$, where $x$ and $y$ depend on the ring width. The area of the multiple cell, using the area of the primitive cell as unit, is given by the determinant

$$
\left[\begin{array}{cc}
f_{u-1} & x \\
-f_{u} & y
\end{array}\right]
$$

It is the number of points in the multiple cell.

Wrapping of the multiple cell is obtained by identification of the translation $\left(f_{u-1},-f_{u}\right)$ to the identity which is the result of gluing of two sides of this cell. The two other sides give the two boundaries of the ring. This type of description shows that the two boundaries are equivalent with the translation $(x, y)$. Counting the number of points in the ring amounts to counting the number of points in the cell, but in the cell the two boundaries are equivalent, so both count for one. Then we have to add to the cell area the number of points on a border, which is $f_{u-1}+f_{u}$. It follows that the number of points in the ring including pentagonal sites inside and heptagonal and hexagonal sites outside, as in Fig. 11, is given by the determinant

$$
\left[\begin{array}{ll}
f_{u-1} & x+1 \\
-f_{u} & y+1
\end{array}\right]
$$

In the example of Fig. 11(a) this is

$$
\left[\begin{array}{cc}
21 & 0 \\
-34 & 10
\end{array}\right]
$$

with $x=-1$ and $y=9$ giving 210 sites in the ring. For Fig. $11(b)$ it is

$$
\left[\begin{array}{cc}
34 & 13 \\
-55 & -5
\end{array}\right]
$$

with $x=12$ and $y=-6$ giving 545 sites in the ring.

\subsection{From boundary to boundary}

It is convenient to refer to rings by the number $u$ in $f_{u}$ corresponding to their most visible parastichy. Their boundaries have $f_{u}+f_{u-1}=f_{u+1}$ points. How is the outside border for $u$ related to the inside border for $u+1$ ? Fig. 12 shows this relation with the example $u=9$ so with $f_{u}=34$ and $f_{u+1}=55$. A chain can be described as a chain of long segments (two heptagonal or pentagonal sites separated by two hexagonal sites) or short segments (two heptagonal or pentagonal sites separated by one hexagonal site). In this example the first

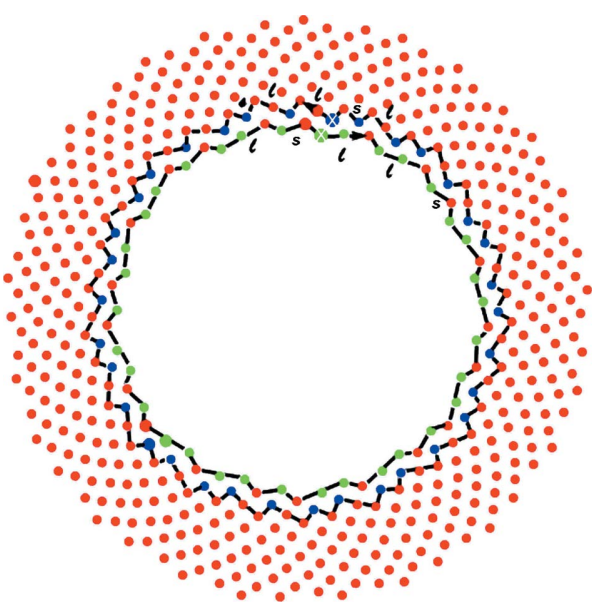

Figure 12

The outside border of the ring with 210 sites and the inside border of the ring with 545 sites. These boundaries are two chains of sites, one with heptagonal sites (green) separated by one or two hexagonal sites (red) having 55 sites, the other with pentagonal sites (blue) separated by hexagonal sites (red) having 89 sites. Starting from the first green site (cross) and rotating as indicated by arrows, we can describe a chain as a succession of long $(l)$ or short $(s)$ segments. Starting from the first blue site (cross) there is also a chain rotating in the reverse orientation. Both chains are related by an inflation symmetry.

chain is coded LLSLLSLSLLSLLSLSLLSLS and the other is coded LLSLLSLSLLSLLSLSLLSLSLLSLLSLSLLSLS, but it is very important to notice that the chains are oriented in order to have an increase of site index when jumping from their first site (first heptagon or pentagon) to the close one corresponding to the positive Fibonacci number $f_{u-1}$ for the first chain or $f_{u}$ for the other. Then the orientation of the two chains is reversed. Nevertheless, as chains are palindromes, using another origin with a circular permutation leads to a symmetric representation.

The inflation law relating the two chains is $L \rightarrow L S$ and $S \rightarrow L$, as usual for Fibonacci chains, but this breaks the symmetry. It is then possible to have a law preserving the palindromic symmetry, but relating a chain defined by $u-1$ to a chain defined by $u+2$. This law, which is $L \rightarrow L S L S L$ and $S \rightarrow L S L$, relates chains of lengths $f_{u}$ and $f_{u+3}$. This law works perfectly well if lengths are even numbers. If lengths are odd numbers, the first two letters obtained this way have to be put at the sequence end to respect symmetry.

It is possible to conclude that the boundaries are related by symmetry operations. Inside a given ring the inner and the outer boundaries are related by some kind of translation, but which appears in the plane as a spiralling transformation coupling a rotation and a radial expansion. Then going to the next ring there is an inflation symmetry associated with a reversal of orientation.

\section{Noble phyllotaxis versus false phyllotaxis}

\subsection{Other values of the parameter $\lambda$ and their approximants}

All the properties we have discussed above are a consequence of the continued fraction expansion of $\lambda$ which gives, 
by truncation, convergent rational approximants (Rivier et al., $1984)$. When $\lambda$ is a noble number, $\left\{q_{1}, q_{2}, \ldots, 1,1,1, \ldots\right\}$ in continued fraction expansion it is possible to extrapolate from $\lambda=1 / \tau=\{1,1,1,1, \ldots\}$. False phyllotaxis is obtained with other rational or irrational $\lambda$, but never appears in nature. It is interesting to follow distances between neighbours (similar to Fig. 9 for true phyllotaxis) as a function of $s$ and see how they evolve from grain to grain. Consider the three distances between a site at $s$ and neighbouring site at $s+\delta s_{i}$ as three functions of $s$ with constant $\delta s_{i}$ characteristic of each grain. These three functions have minimum values which are different, so leading to a dispersion of neighbour distances. Going from grain to grain with new sets of $\delta s_{i}$ two minima increase but there is a smallest distance with a minimum decreasing with $s$. Voronoi cells, even if they remain hexagons in grains, become more and more elongated with increasing $s$, leading to a spiderweb aspect. This is the signature of the non-uniformity associated with non-noble numbers.

In the case of a true phyllotaxis we have seen in $\$ 3.2$ that neighbours are gathered on $f_{u}$ Fermat spirals (parastichies) when they are separated by $\delta s=f_{u}$. This is because convergent approximants of $\lambda=1 / \tau$ obtained by truncation of its continued fraction expansion have the form $f_{u-1} / f_{u}$. In the case of non-noble numbers, the truncation of the continued fraction expansion of $\lambda$ at $q_{u}$ leads to a principal rational approximant $\left\{\lambda^{\prime}=r_{u} / p_{u} ; r_{u}, p_{u} \in \mathbb{Z}\right\}$. Then some neighbours are separated by $\delta s=p_{u}$ and gathered on $p_{u}$ similar spirals. Successive $p_{u}$ and $r_{u}$ are related by

$$
p_{u}=q_{u} p_{u-1}+p_{u-2} .
$$

In a grain consider a Delaunay triangle of close neighbours. The three edges of the triangle define three directions of parastichies in the grain. The homogeneity of the grain requires that these three families of parastichies, characterized by three index separations $\left\{\delta s_{1}, \delta s_{2}, \delta s_{3}\right\}$, are all the same in the grain. If the three separations are given in increasing order, the closure of edges of the triangle results in the triangular relation $\delta s_{3}=\delta s_{1}+\delta s_{2}$. If, in a grain, a site $s$ is neighbouring a site $s+p_{u}$, the triangular relation and equation (6) are incompatible except if $q_{u}=1$ (as it is for all not too small $u$ in true phyllotaxis). To solve this incompatibility new intermediate approximants appear: to $r_{u} / p_{u}$ are associated $q_{u}$ approximants $r_{u}^{(i)} / p_{u}^{(i)}$ with the sub-index $(i) \in\left[1, q_{u}\right]$. The integers $p_{u}^{(i)}$ and $r_{u}^{(i)}$ are defined by $p_{u+1}^{(1)}=p_{u-1}+p_{u}$ and $p_{u+1}^{(i)}=p_{u+1}^{(i-1)}+p_{u}$, so we can write $p_{u+1}^{(0)}=p_{u-1}$ and $p_{u+1}^{\left(q_{u}\right)}=p_{u+1}$. Using denominators of these intermediate approximants it is then possible to satisfy the triangular relation with the separations. In this case at least one principal approximant denominator $p_{u}$ appears in this list, and it appears in $q_{u}+2$ successive grains.

Notice that distances between sites separated by a $\delta s$ related to an approximant $r_{u}^{(i)} / p_{u}^{(i)}$ are given by a similar relation to equation (3) replacing $f_{u-1}$ and $f_{u}$ by $r_{u}^{(i)}$ and $p_{u}^{(i)}$. This can be done also in order to have minimum values using equation (4).

\subsection{Phyllotaxis with a noble number}

Phyllotaxis is characterized by two parameters. One, $\lambda$, describes the azimuthal (circle) map $\theta(s+1)=\theta(s)+2 \pi \lambda$, independently of the radial (growth) map $r(s)$. The radial map defines the generative spiral $r(\theta)$. On a flat substrate, the parameter $\alpha$ describes all kinds of parabolic spirals $r(\theta)=a \theta^{\alpha}$, but also the equiangular spiral $r(\theta)=a \exp \theta(\alpha=\infty)$ and the dense spiral $r(\theta)=a \ln \{1+\theta\} \quad(\alpha=0)$ (Rivier \& Goldar, 1998). On a curved substrate, the spiral is defined by projection and an effective growth parameter $\alpha>0$ can be defined, with $\alpha_{0}=1 / 2$ for a flat substrate, $\alpha>\alpha_{0}$ and a looser spiral for a substrate with positive curvature, and $\alpha>\alpha_{0}$ and a tighter spiral for a substrate with negative curvature (our next paper). In this paper, we concentrate on the 'ideal' flat case, $\lambda=1 / \tau$, $\alpha_{0}=1 / 2$ that yields a sequence of concentric circular grain boundaries.

An infinite phyllotactic structure can be obtained from any $0<\lambda>1$ that is a noble irrational, $\lambda=\left\{q_{1}, q_{2}, \ldots, q_{u}, 1 / \tau\right\}$, beginning with an arbitrary rational $\left\{q_{1}, q_{2}, \ldots, q_{u}\right\}$ and terminating with a golden tail $1 / \tau=\{1,1, \ldots, 1, \ldots\}$ (continuous fraction expansion with $q_{i} \geq 1$ ). Now, all rationals between 0 and 1 can be arranged and ordered on a Farey tree, and the golden tail oscillates down the tree starting from its defining rational $\left\{q_{1}, q_{2}, \ldots, q_{u}\right\}$, leading to a cascade of regular (parastichy) transitions through grain boundaries (Rothen \& Koch, 1989a; Rivier et al., 1991; Levitov, 1991; Rivier, 1992; Douady, 1998; Koch et al., 1998). Note that the topological mechanism is always the same, articulated through the golden mean $1 / \tau=\{1,1, \ldots, 1, \ldots\}$. The actual value of the parameter $\lambda$ also oscillates between narrower bounds as the structure becomes larger. This implies also that the measurement of $\lambda$ from a given, finite structure is not that precise, and that it is collimated as the structure grows in size. Note that there are no exceptions in nature (i.e. no false phyllotaxis). Such a tree already appears in Koch's thesis and in Rothen \& Koch (1989b), but the remark that it was isomorphic to Farey is due to Rivier et al. (1991). It was made independently by Levitov (1991). Regular and singular transitions were first introduced by Koch and Rothen. In Levitov, the singular transition branches are even disconnected (as an energy flow) from the main Farey tree (Lee \& Levitov, 1998).

The corresponding structure has a more or less disorganized central core moulded by the starting rational $\left\{q_{1}, q_{2}, \ldots, q_{u}\right\}$, followed by the well established phyllotactic structure moulded by the golden tail. Obviously, the structure must be (much) larger than its core to exhibit it. Note that even for $\lambda=1 / \tau$ there is an apparent core for $s \leq 30$ within which successive grain boundaries touch or even overlap. Topologically, the structure is perfect down to $s=1$ (Table 1). Overlap occurs on a strongly negative substrate (our next paper).

False phyllotaxis examples exhibit, beyond their core, spiderweb structures, generated by $\lambda$ that are either rationals, $\lambda=\left\{q_{1}, q_{2}, \ldots, q_{u}, \infty\right\}, \quad$ or Liouville irrationals, $\quad \lambda=$ $\left\{q_{1}, q_{2}, \ldots, q_{u}, Q, \ldots\right\}$, with the integer $Q \gg q_{i} \geq 1$. On the Farey tree, the Liouville irrational goes through a large number $\leq Q$ of singular transitions. 


\section{Conclusions}

An infinite number of sites at distance $d$ organized on the nodes of a triangular tiling defined by a hexagonal lattice have the translational and rotational symmetries of this lattice. With each node it is possible to associate a hexagon considered as its Voronoi cell. This tessellation with regular hexagonal cells obeys the Euler relation $F-E+V=0$ where $F, E$ and $V$ are, respectively, the number of faces, edges and vertices in the tiling. If we consider points occupying a finite domain limited by a circular boundary, the Euler relation becomes $F-E+V=1$ and non-hexagonal cells appear among the hexagonal cells. The distribution of points in the disc therefore contains inherent defects and is no longer invariant under the symmetry operations of classical crystallography.

The algorithm of phyllotaxis builds a dense organization of points in a disc which optimizes the homogeneity of the area associated with each point and the isotropy of the environment of each point in a situation of circular symmetry. To identify the laws specific to this type of organization we have described it using Voronoi and Delaunay decompositions which make apparent the presence of intrinsic defects. These defects are pentagonal and heptagonal cells associated two by two in dipoles organized along narrow concentric rings separating larger rings containing hexagonal cells continuously deformed. Such an organization can be understood by considering two types of disorder in the organization: a metric disorder corresponding to fluctuations of distances between first neighbours, as the hexagonal cells are not regular, and a topological disorder corresponding to the presence of pentagonal and heptagonal cells associated into dipoles (dislocations). These two disorders interact to build the organization. Notice that, as usual, dislocations are called topological defects, but the notion of defect refers to the perfect crystalline structure. In phyllotaxis pentagon-heptagon dipoles are basic constituents of the structure.

The role of pentagonal and heptagonal cell dipoles is to introduce rows of new cells needed to maintain the homogeneity of area when the radius of the structure increases, as does a dislocation in metallurgy. This organization is also reminiscent of grains and grain boundaries of dislocations in metals, but here the grain boundaries are imposed by the circular symmetry whereas, in metal grain boundaries, they result from processing treatments. Finally, a very specific order is observed in the organization of these dipoles in concentric grain boundaries: the radius of the grain boundaries, or the width of grains, follows the Fibonacci series, while the sequence of dipoles along the grain boundaries is that of onedimensional quasicrystalline sequences which are deduced from each other by an inflation-deflation rule which leads to a self-similar structure.

Thus the topological constraint of the circular symmetry introduces an original inflation-deflation symmetry replacing the translational and rotational symmetries of classical crystallography. Still using the language of metallurgy, the Voronoi cell and parastichies could be seen as the repeat unit and the crystallographic lattice planes of this unconventional structure.

Rings of dipoles, the grain boundaries, with their self-similar organization, are the fundamental characteristic of phyllotaxis. We are investigating phyllotactic tilings on the sphere and on the hyperbolic plane, always keeping a constant area for each site. The geometry of the dipole rings and their succession is the same as on the flat disc. The effect of the Gaussian curvature of the tiled surface only concerns the width of the hexagonal grains. So whatever is the underlying geometry, grain boundaries are structurally blocked, without any adjustable parameter. This is true from a topological but also a metric point of view.

The organization of the florets of the flowers of many plants is the earliest known example of the implementation of the algorithm of phyllotaxis, and it was indeed at the origin of the development of this domain of studies. As already quoted in $\S 1$, recent works on purely physical systems offer new examples (Rivier et al., 1984; Douady \& Couder, 1996; Yoshikawa et al., 2010).

All these examples are macroscopic assemblies of elements such as florets, leaves, convection cells, ferrofluid droplets and bubbles. They have in common not only a circular symmetry, but also the ability of their elements to move with respect to each other in order to adjust their distribution in the course of the growth process. This symmetry and behaviour are also presented at the microscopic level by molecular associations of soft condensed matter and biological materials. Their molecular interactions allow an internal mobility and their interfacial tension in solution imposes the circular symmetry so that they could be considered by a similar approach. This is why we proposed it for describing collagen fibres (Charvolin \& Sadoc, 2011). If this were so, whereas in the cases of flowers or droplets the growth is generated from the centre and in that of the convection cells the organization builds itself at a collective transition, this growth of fibrils by addition at their surface would provide a third example of the power of the algorithm of phyllotaxis in structural studies.

\section{References}

Adler, I. (1998). J. Algebra, 205, 227-243.

Bravais, A. \& Bravais, L. (1837). Ann. Sci. Nat. (second series), 7, 42110.

Charvolin, J. \& Sadoc, J.-F. (2011). Biophys. Rev. Lett. 6, 13-27.

Coxeter, H. S. M. (1961). Introduction to Geometry. New York: Wiley. Coxeter, H. S. M. (1972). J. Algebra, 20, 167-175.

Douady, S. (1998). Symmetry in Plants, edited by R. V. Jean \& D. Barabé, pp. 335-358. Singapore: World Scientific.

Douady, S. \& Couder, Y. (1992). Phys. Rev. Lett. 68, 2098-2101.

Douady, S. \& Couder, Y. (1996). J. Theor. Biol. 178, 255-312.

Gonzàlez, A. (2010). Math. Geosci. 42, 49-64.

Jean, R. V. (1983). Math. Biosci. 64, 1-27.

Jean, R. V. (1992). Five-fold Symmetry, edited by I. Hargittai. Singapore: World Scientific.

Koch, A.-J., Bernasconi, G. \& Rothen, F. (1998). Symmetry in Plants, edited by R. V. Jean \& D. Barabé, pp. 459-486. Singapore: World Scientific. 
Lee, H. W. \& Levitov, L. S. (1998). Symmetry in Plants, edited by R. V. Jean \& D. Barabé, pp. 619-653. Singapore: World Scientific.

Levitov, L. S. (1991). Europhysics Lett. 14, 533-539.

Ridley, I. N. (1982). Math. Biosci. 58, 129-139.

Rivier, N. (1986). J. Phys. France, 47, C3-299-C3-309.

Rivier, N. (1988). Mod. Phys. Lett. B, 2, 195-206.

Rivier, N. (1992). J. Phys. Condens. Matter, 4, 913-943.

Rivier, N. \& Goldar, A. (1998). Aperiodic '97, edited by M. de Boissieu, J. -L. Verger-Gaugry \& R. Currat, pp. 131-135. Singapore: World Scientific.

Rivier, N., Koch, A.-J. \& Rothen, F. (1991). Biologically Inspired Physics, edited by L. Peliti, pp. 371-380. New York: Plenum.

Rivier, N., Miri, M. F. \& Oguey, C. (2005). Colloids Surf. A Physicochem. Eng. Asp. 263, 39-45.
Rivier, N., Occelli, R., Pantaloni, J. \& Lissowski, A. (1984). J. Phys. France, 45, 49-63.

Rothen, F. \& Koch, A.-J. (1989a). J. Phys. France, 50, 633-657.

Rothen, F. \& Koch, A.-J. (1989b). J. Phys. France, 50, $1603-$ 1621.

Rothen, F., Pieranski, P., Rivier, N. \& Joyet, A. (1993). Eur. J. Phys. 14, 227-233.

Thompson, D. W. (1917). On Growth and Form, 2nd ed., ch. XIV, pp. 912-933. Cambridge University Press.

Turing, A. M. (1992). In Morphogenesis. Collected Works of A. M. Turing, edited by P. T. Saunders. Amsterdam: North-Holland.

Yeatts, F. R. (1997). Math. Biosci. 144, 71-81.

Yoshikawa, H. N., Mathis, C., Maissa, P., Rousseau, G. \& Douady, S. (2010). Eur. Phys. J. E, 33, 11-25. 